\title{
Discovery of a highly magnetic He-sdO star from a double-degenerate binary merger
}

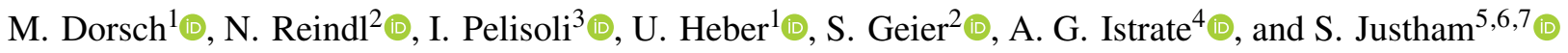 \\ 1 Dr. Karl Remeis-Observatory \& ECAP, Friedrich-Alexander University Erlangen-Nürnberg, Sternwartstr. 7, 96049 Bamberg, \\ Germany \\ e-mail: matti.dorsch@fau.de \\ 2 Institut für Physik und Astronomie, Universität Potsdam, Haus 28, Karl-Liebknecht-Str. 24/25, 14476 Potsdam-Golm, Germany \\ 3 Department of Physics, University of Warwick, Gibbet Hill, Road, Coventry CV4 7AL, UK \\ 4 Department of Astrophysics/IMAPP, Radboud University, PO Box 9010, 6500 GL Nijmegen, The Netherlands \\ ${ }^{5}$ University, and National Observatory, of the Chinese Academy of Sciences, Beijing 100012, PR China \\ 6 Anton Pannekoek Institute for Astronomy \& GRAPPA, University of Amsterdam, Postbus 94249, 1090 GE Amsterdam, \\ The Netherlands \\ 7 Max-Planck-Institut für Astrophysik, Karl-Schwarzschild-Straße 1, 85741 Garching, Germany
}

Received 10 December 2021 / Accepted 17 January 2022

\begin{abstract}
Helium-rich hot subdwarf stars of spectral type $\mathrm{O}(\mathrm{He}-\mathrm{sdO})$ are considered prime candidates for stellar merger remnants. Such events should lead to the generation of strong magnetic fields. However, no magnetic He-sdO has yet been unambiguously discovered despite the high magnetic rate $(20 \%)$ among white dwarf stars, the progeny of hot subdwarfs. Here we present the discovery of a strong magnetic field $(B=353 \pm 10 \mathrm{kG})$ from Zeeman-split hydrogen, helium, and metal lines in the optical X-shooter spectrum of an $\mathrm{He}$-sdO and present the first spectroscopic analysis of any magnetic hot subdwarf. For this we used line-blanketed TLUSTY nonlocal thermodynamic equilibrium models and assumed a simple homogeneous magnetic field. The derived atmospheric parameters $T_{\text {eff }}=44900 \pm 1000 \mathrm{~K}$ and $\log g=5.93 \pm 0.15$ are typical for He-sdO stars, while the star is less hydrogen-poor than most He-sdOs at $\log n(\mathrm{He}) / n(\mathrm{H})=+0.28 \pm 0.10$. The star is a slow rotator $\left(v_{\mathrm{rot}} \sin i<40 \mathrm{~km} \mathrm{~s}^{-1}\right)$. Its chemical composition is N-rich and C- and $\mathrm{O}$-poor, and the $\mathrm{Si}$ and $\mathrm{S}$ abundances are close to solar. Combining the atmospheric parameters with Gaia parallax and photometry, the stellar radius and luminosity are found to be typical for He-sdOs and place the star on the helium main sequence in the HertzsprungRussell diagram. Its mass of $0.93_{-0.30}^{+0.44} M_{\odot}$, although uncertain, appears to be remarkably high. The strong magnetic field along with the atmospheric parameters and metal abundances provide overwhelming evidence for the double-degenerate merger scenario.
\end{abstract}

Key words. stars: magnetic field - subdwarfs - stars: early-type

\section{Introduction}

Most hot subdwarf-O (sdO) and subdwarf-B (sdB) stars are helium-burning stars with hydrogen envelopes that are too thin to sustain hydrogen shell burning (Heber 2009, 2016). The bulk of sdB stars form the hot extreme of the horizontal branch at masses close to half solar and radii of $0.1-0.2 R_{\odot}$. Various types of binary interaction can explain the majority of hot subdwarf stars (Pelisoli et al. 2020). This includes stable Roche overflow to a main sequence companion star, common-envelope ejection by white dwarf (WD) companions to red giant stars, and the merging of two helium-core white dwarfs (He-WDs; Han et al. 2002, 2003; Webbink 1984). Because hot subdwarf stars have very thin envelopes, they evolve directly to the WD sequence without an excursion to the asymptotic giant branch. A smoking gun for the merger scenario would be the detection of strong magnetic fields, which are predicted by detailed merger models for massive stars (Schneider et al. 2019) and carbon-oxygen (CO) WD mergers (Ji et al. 2013; Zhu et al. 2015) and are likely to occur for helium WD mergers as well.

About $20 \%$ of all WD stars within $20 \mathrm{pc}$ of Earth are known to host surface magnetic fields, with strengths ranging from a few kilogauss to several hundred megagauss (e.g.
Bagnulo \& Landstreet 2020, 2021). In contrast, magnetic fields have not yet been directly observed for any hot subdwarf star. Spectropolarimetry has failed to provide evidence for the magnetic fields of more than $40 \mathrm{sdB}$ and $\mathrm{sdO}$ stars, with upper limits of about 1 to $2 \mathrm{kG}$ (Landstreet et al. 2012; Mathys et al. 2012; Randall et al. 2015; Bagnulo et al. 2015). Indirect signs of magnetism have been claimed from light and radial velocity variations tentatively attributed to magnetic spots (Jeffery et al. 2013; Geier et al. 2015; Balona et al. 2019; Momany et al. 2020; Vos et al. 2021), but lack independent confirmation.

A first helium-rich hot O-type subdwarf (He-sdO) star with a significant magnetic field $(\approx 300-700 \mathrm{kG})$ was identified spectroscopically by Heber et al. (2013), although this star has not been analysed to date. Here we present the first spectroscopic analysis of a hot subdwarf star with a strong magnetic field, the He-sdO Gaia DR2 5694207034772278400 (henceforth J08092627).

\section{Observations}

J0809-2627 was identified as a candidate hot subdwarf by Geier et al. (2019). We obtained follow-up spectroscopy with the Intermediate Dispersion Spectrograph (IDS) spectrograph at the 


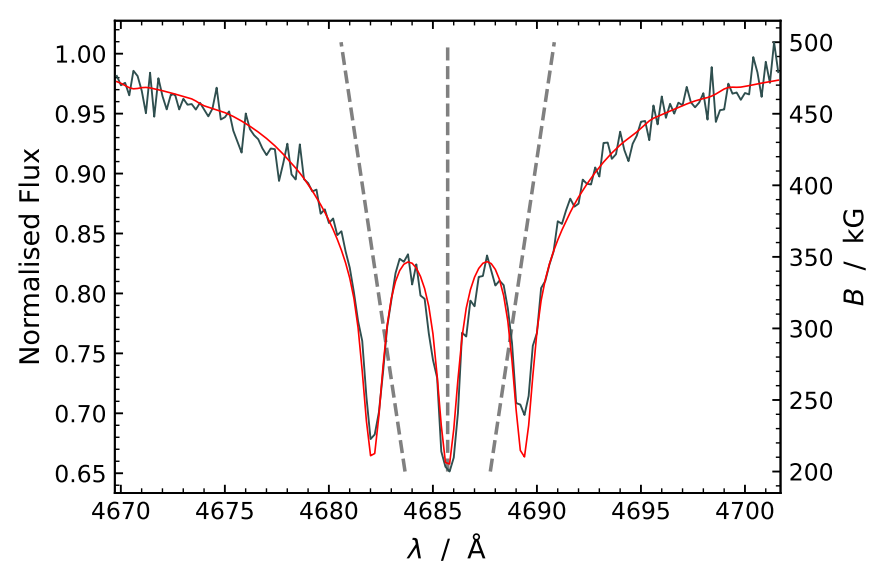

Fig. 1. Zeeman-split He II $4686 \AA$ line in the radial-velocity-corrected $\mathrm{X}$-shooter spectrum of J0809-2627 (grey). The best-fit model is shown in red. The dashed lines illustrate the positions of the three components that depend on the magnetic field strength due to the linear Zeeman effect.

Isaac Newton Telescope (INT) to confirm this classification and identified the star as a helium-rich sdO star. At a resolution of $\Delta \lambda \approx 4 \AA$ and signal-to-noise ratio ( $\mathrm{S} / \mathrm{N}$ ) of 50, the IDS spectrum shows strongly broadened hydrogen and helium lines, which we tentatively interpreted as rapid rotation.

Because hot subdwarf stars are known to be slow rotators (Geier \& Heber 2012), the strong line-broadening of J0809-2627 is unusual. Therefore, the star was observed again in April 2021 with the medium-resolution spectrograph X-shooter at the ESOVLT. We used the 3070-7400 $\AA$ range of the UVB and VIS channels, which covers all relevant spectral lines at a mean $\mathrm{S} / \mathrm{N}$ of about 80 and at a resolving power of $R=\lambda / \Delta \lambda \approx 10000$. At this resolution, which is eight times better than that of IDS, strong Zeeman-split multiplets are clearly visible, demonstrating that the line broadening is not due to rapid rotation but is caused by the presence of a magnetic field (see Fig. 1). The 3070-7400 A spectral range is shown in Fig. D.1. All exposure times can be found in Appendix A.1.

The light curve of J0809-2627 has been observed by the TESS satellite but does not show significant variability at $\mathrm{a} \approx 0.42$ parts per thousand (ppt) detection limit (see Appendix A.2). However, J0809-2627 is located in a relatively crowded field, so contamination by nearby stars may hide any variability.

\section{Models}

We used a grid of atmospheric structures computed with the TLUSTY code as the basis of our spectroscopic analysis. A description of the code is given in Hubeny \& Lanz (2017a,b,c). These models are plane-parallel, homogeneous, hydrostatic, and include $\mathrm{H}, \mathrm{He}, \mathrm{C}, \mathrm{N}, \mathrm{O}, \mathrm{Si}, \mathrm{P}, \mathrm{S}, \mathrm{Fe}$, and $\mathrm{Ni}$ in non-local thermodynamic equilibrium.

The observed line splittings can be explained by the linear Zeeman effect caused by the presence of a strong surface magnetic field. The magnetic field was assumed to be homogeneous across the visible surface, that is, of uniform strength and direction. Its axis was allowed to be inclined at an angle $\psi$ with respect to the line of sight. Smaller inclinations lead to stronger displaced Zeeman components relative to the central component. The magnetic field in J0809-2627 is not strong enough to have a large effect on the atmospheric structure beyond the additional metal line opacity in the UV region (Wickramasinghe \& Martin 1986; Tremblay et al. 2015) ${ }^{1}$. In addition, the linear Zeeman effect dominates over the quadratic Zeeman effect at strengths below about $1 \mathrm{MG}$ (Garstang \& Kemic 1974). Therefore, our approach was to consider only the linear Zeeman effect and only in the spectrum synthesis. We modified the spectrum synthesis code SYNSPEC (Hubeny \& Lanz 2017a) to include linear Zeeman multiplets for hydrogen, helium, and detectable metal lines, similar to the method Kawka \& Vennes (2011) used to model the cool magnetic WD NLTT 10480, which has a magnetic field strength $(\approx 500 \mathrm{kG})$ similar to that of J0809-2627. Polarised radiative transfer in the lines was not included in our simple models. More details of our model for the magnetic field are given in Appendix B.

\section{Spectral fits}

We performed a global $\chi^{2}$ fit to the X-shooter spectrum of J08092627 to determine its atmospheric parameters, including $B$ and $\psi$. Some of the strongest hydrogen and helium lines that could be used for the fit are shown in Fig. 2. The $\chi^{2}$ fit prefers a projected rotational velocity of $v_{\text {rot }} \sin i=34 \mathrm{~km} \mathrm{~s}^{-1}$. However, $v_{\text {rot }} \sin i$ is not well constrained because broadening of the displaced Zeeman components may also be caused by a more complex magnetic field geometry. Possibly for the same reason, the central components of several helium lines are sharper in the observation than predicted by our model. The strength of the central components of Zeeman triplets may also be somewhat increased by magneto-optical effects (Martin \& Wickramasinghe 1981), which are not included in our models. Most observed metal lines appear sharp given the limited resolution of the X-shooter spectra. The final atmospheric parameters as derived from the $\mathrm{X}$-shooter spectra are listed in the left part of Table 1. All uncertainties are estimated because they are dominated by systematic effects rather than by noise.

As demonstrated in Fig. 3, the X-shooter spectrum of J08092627 shows strong N III-IV, Si IV, and S IV lines. The determination of abundances was complicated not only by the magnetic field but also because most transitions originate from high-lying energy levels that are hard to model, even in non-magnetic sdO stars. This meant that some predicted lines could not be used for the abundance determination, for example the C III triplet at $4070 \AA$ and N III $4379 \AA$. Metal abundances were therefore estimated by eye, by comparing the observed spectra with models with different abundances and keeping the atmospheric parameters fixed to the best-fit values. The derived abundances are listed on the right side of Table 1. J0809-2627 is enriched in nitrogen at close to 20 times the solar abundance (by number). Carbon and oxygen seem to be at least somewhat subsolar because no lines from these elements are clearly detected - in particular, the C III $4159 \AA$, C IV $5805 \AA$, and O III $3760 \AA$ multiplets. The photosphere therefore likely consists of material processed by hydrogen fusion in the CNO cycle. Silicon and sulphur are enriched at about two and six times their solar value, respectively.

The X-shooter spectra give us a precise radial velocity for J0809-2627. To test whether J0809-2627 is radial velocity

\footnotetext{
1 Although iron and nickel lines are not observed in the optical spectrum of J0809-2627, they were still included in our models at abundances of three times their solar value. This is necessary because the opacity of Zeeman-spilt iron group lines in the UV region likely has a significant effect on the atmospheric structure. For the same reason, we used a microturbulent velocity of $6 \mathrm{~km} \mathrm{~s}^{-1}$ for the atmospheric structure calculations only.
} 
M. Dorsch et al.: Discovery of a highly magnetic He-sdO
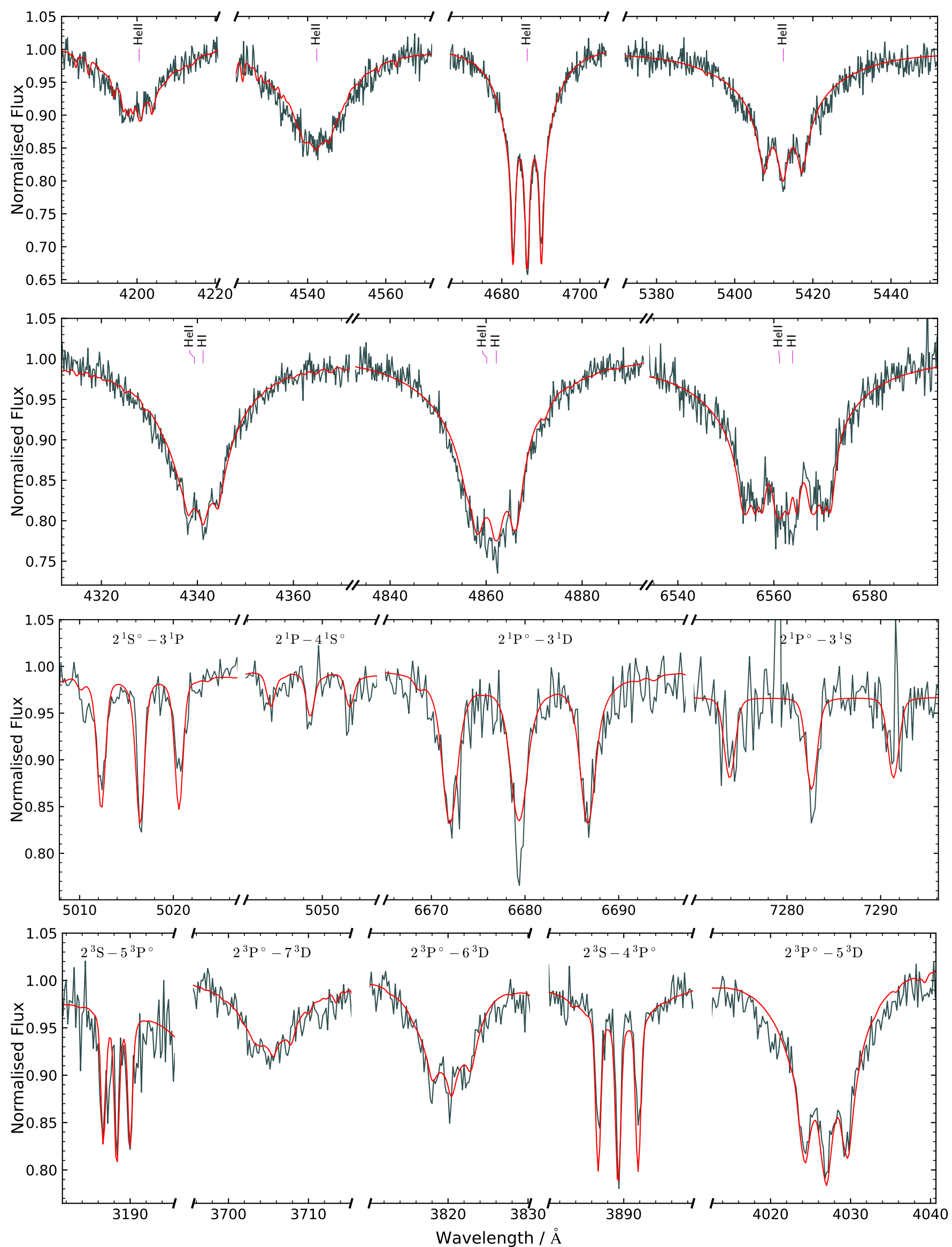

Fig. 2. Strongest He II and HI lines, as well as the best-fitting He I lines in the X-shooter spectrum of J0809-2627 (grey). The best-fit model is shown in red. Labels indicate He II and H I line positions at $B=0$, as well as lower and upper LS terms for He I. 
Table 1. Atmospheric parameters derived from spectroscopy.

\begin{tabular}{lccccc}
\hline \hline & Spectral fit & & & Element & $\log n_{\mathrm{X}} / \sum_{i} n_{i}$ \\
\cline { 1 - 2 } \cline { 5 - 6 }$T_{\text {eff }}(\mathrm{K})$ & $44900 \pm 1000$ & & $\mathrm{H}$ & $-0.46 \pm 0.07$ \\
$\log g$ & $5.93 \pm 0.15$ & & $\mathrm{He}$ & $-0.18 \pm 0.03$ \\
$\log n(\mathrm{He}) / n(\mathrm{H})$ & $+0.28 \pm 0.10$ & & $\mathrm{C}$ & $<-3.74^{+0.40}$ \\
$B(\mathrm{kG})$ & $353 \pm 10$ & & $\mathrm{~N}$ & $-2.98 \pm 0.20$ \\
$\psi\left({ }^{\circ}\right)$ & $64 \pm 25$ & & $\mathrm{O}$ & $<-3.92^{+0.30}$ \\
$v_{\text {rad }}\left(\mathrm{km} \mathrm{s}^{-1}\right)$ & $33 \pm 2$ & & $\mathrm{Si}$ & $-4.26 \pm 0.30$ \\
$v_{\text {rot }} \sin i\left(\mathrm{~km} \mathrm{~s}^{-1}\right)$ & $<40$ & & $\mathrm{~S}$ & $-4.16 \pm 0.30$ \\
\hline
\end{tabular}

Notes. Elemental abundance results are stated by number fraction. Upper limits are given as best-fit values, while their uncertainties represent values that can clearly be excluded.

variable or not, follow-up spectroscopic observations are necessary because the IDS spectra are of insufficient quality.

By combining the spectroscopic surface gravity, the Gaia parallax, and the spectral energy distribution (SED), the mass, radius, and luminosity of J0809-2627 can be derived. This analysis is described in Appendix C. While the stellar radius, $0.184 \pm 0.011 R_{\odot}$, and luminosity, $123_{-16}^{+19} L_{\odot}$, are typical for HesdO stars, the derived mass for J0809-2627, $0.93_{-0.30}^{+0.44} M_{\odot}$, is rather high. It should be noted that the uncertainty of the mass is considerable due the limited accuracy of the spectroscopic surface gravity. These parameters place J0809-2627 close to the zero-age helium main sequence at $\approx 0.8 M_{\odot}$ (Paczyński 1971), which means it consists of a helium core with next to no hydrogen envelope left.

\section{Evolutionary status}

In general, the presence of a strong magnetic field can be considered the smoking gun for stellar mergers (Schneider et al. 2019). Given that J0809-2627 is an apparently single magnetic helium-rich sdO star and likely more massive than usual for that class of star (Schindewolf et al. 2018; Dorsch et al. 2019, 2020, 2021), the merging of two He-WD stars can be considered a natural scenario for its formation. In this scenario an object with a mass as high as $0.93 M_{\odot}$ would be unlikely, but the scenario is predicted to produce significant numbers of HesdO stars with masses of up to about $0.8 M_{\odot}$ (Han et al. 2003), which would be well within the uncertainty of our mass for J0809-2627. A related formation channel for He-sdO stars is the merger of an He-WD with a post-sdB WD, which would produce a hybrid WD with a CO core and a thick helium envelope, a so-called HeCO-WD (Justham et al. 2011, following pioneering models from Iben 1990). This channel is a natural consequence of one of the common-envelope formation channels for sdB stars described by Han et al. (2002) and seems likely to contribute substantially to the population of single He-sdO stars. Moreover, the inferred effective temperature and surface gravity of J08092627 are close to those of the densest region of the population predictions in Justham et al. (2011).

Based on the three-dimensional simulations of CO WD mergers of Yoon et al. (2007) and Lorén-Aguilar et al. (2009), García-Berro et al. (2012) showed that strong magnetic fields can be generated in the hot, convective, differentially rotating corona that surrounds the primary during and shortly after the merging. These magnetic fields are predicted to be frozen to the outer layers of the final merger product. Magneto-dipole radiation rapidly spins down the newly formed magnetic WD if mag- netic and rotation axes are not aligned. These predictions appear to be consistent with the recent results of Bagnulo \& Landstreet (2021), who found that young WDs with fields exceeding $1 \mathrm{MG}$ are more massive than canonical WDs.

The CNO-processed chemical composition and the relatively slow projected rotation of J0809-2627 provide additional important constraints on merger models. Evolutionary calculations for mergers of He-WDs with He-WDs were performed by Zhang \& Jeffery (2012), Hall \& Jeffery (2016), and Schwab (2018). Zhang \& Jeffery (2012) predict nitrogen-rich surfaces for slow double He-WD mergers, similar to what we find for J0809-2627. Such systems retain the initial composition of the secondary He-WD, which includes a CNO-cycle signature, as observed for J0809-2627. Because our upper limit for the carbon abundance of J0809-2627 is only slightly subsolar, the composite merger model of Zhang \& Jeffery (2012) cannot be excluded for remnant masses below about $0.7 M_{\odot}$. This model assumes that more than half of the secondary mass is rapidly accreted and forms a hot corona, after which the remaining mass is accreted slowly. As shown in Fig. 4, the $0.8 M_{\odot}$ slow merger model of Zhang \& Jeffery (2012) matches the effective temperature and luminosity of J0809-2627 well at the onset of core helium burning.

For hybrid WD mergers, it seems plausible that surface abundances consistent with J0809-2627 are also possible as long as the He-WD is disrupted rather than the hybrid HeCO-WD (in other words, as long as the He-WD is the less massive component of the merging binary). Of the hybrid merger models in Justham et al. (2011), the luminosity and surface temperature of J0809-2627 are matched by mergers in which a hybrid WD of only $0.35 M_{\odot}$ accretes $0.15-0.3 M_{\odot}$ from a merger with a He-WD. Those models are also consistent with the surface gravity of J0809-2627. However, only the more massive of those merger models are within the mass range we infer for J0809-2627.

The best-fit hydrogen abundance for J0809-2627 is higher than that observed for most (extreme) He-sdO stars (Stroeer et al. 2007; Schindewolf et al. 2018). Hydrogen was not included in the merger models of Justham et al. (2011) or Zhang \& Jeffery (2012), while both hydrogen and helium were included in the models of Schwab (2018). The Schwab (2018) models predict that the surface hydrogen abundance depends on the rotational velocity after the merging - models that include rotation result in very hydrogen-poor surfaces, which is not what we find for J0809-2627. Specifically, Schwab (2018) predict rotational velocities of about $30 \mathrm{~km} \mathrm{~s}^{-1}$ once a $0.5 M_{\odot}$ merger remnant reaches the core-helium-burning phase, which would be consistent with the elemental abundance pattern of J0809-2627. However, a more massive $0.7 M_{\odot}$ merger remnant is predicted to be a fast rotator, with $v_{\text {rot }} \approx 100 \mathrm{~km} \mathrm{~s}^{-1}$. This would require a low inclination for J0809-2627 given its low projected rotational velocity $\left(<40 \mathrm{~km} \mathrm{~s}^{-1}\right)$. Alternatively, a misalignment of rotation and magnetic axes might provide further loss of angular momentum through magneto-dipole radiation (García-Berro et al. 2012), which was not included in the calculations of Schwab (2018). In the $0.7 M_{\odot}$ model of Schwab (2018), the surface is predicted to be enriched in carbon, which, however, is not observed for J0809-2627.

J0809-2627 will evolve through a helium-shell-burning phase and eventually become a WD. If the magnetic flux is conserved until J0809-2627 reaches the WD stage $\left(B \sim R^{-2}\right)$, its surface field strength will reach about $120 \mathrm{MG}$ at a radius of $0.01 R_{\odot}$. 


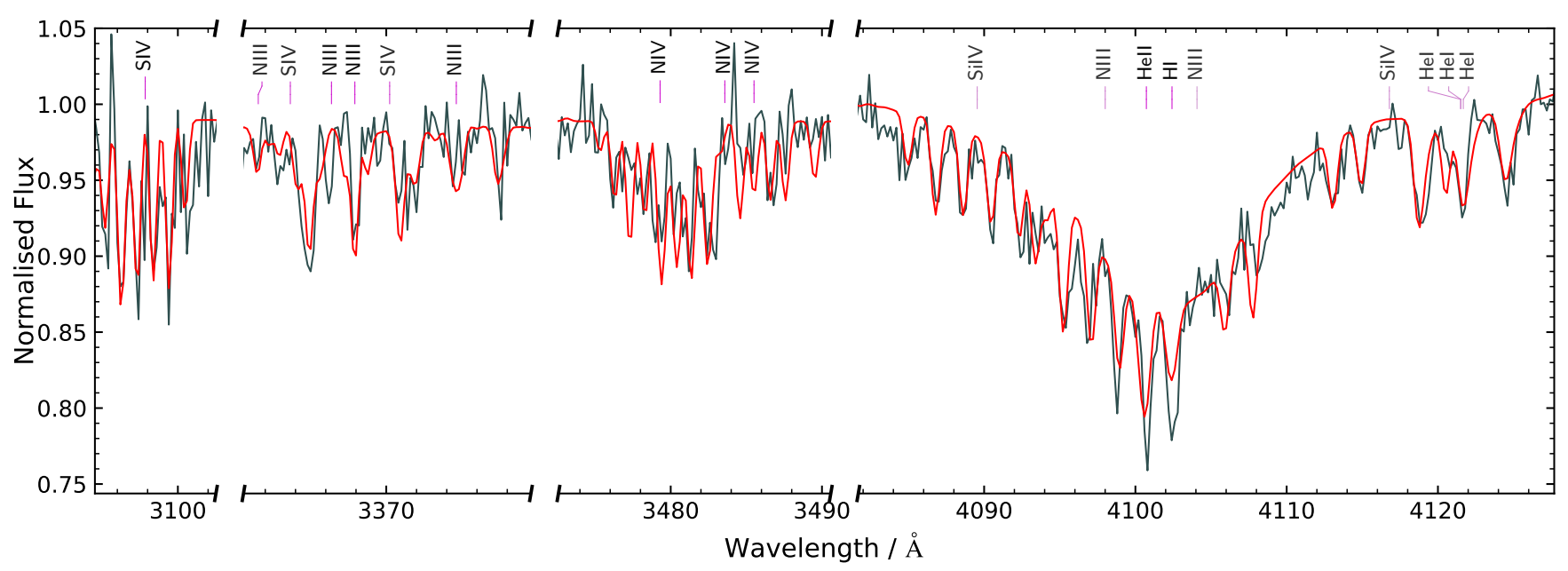

Fig. 3. Zeeman-split N III-IV, Si IV, and S IV lines in the X-shooter spectrum (grey) and the best-fit model (red) of J0809-2627. The projected rotational velocity is set to $v_{\text {rot }} \sin i=20 \mathrm{~km} \mathrm{~s}^{-1}$. Line positions at zero magnetic field are labelled.

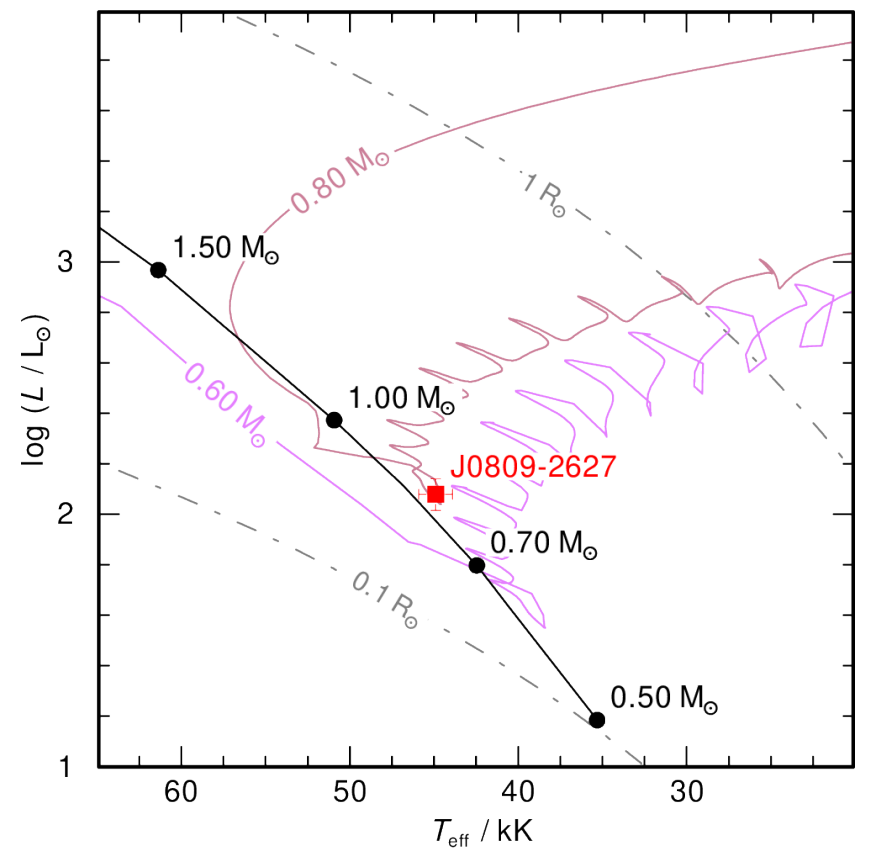

Fig. 4. Position of J0809-2627 (red) in the Hertzsprung-Russell diagram compared to several evolutionary tracks. Pink lines show two double He-WD slow-merger tracks from Zhang \& Jeffery (2012) for metallicity $Z=0.02$. The final masses for both tracks are labelled. Black lines show the zero-age helium main sequence from Paczyński (1971), where black filled circles indicate masses of $0.5,0.7,1.0$, and $1.5 M_{\odot}$. The dashed-dotted grey lines indicate radii of 0.1 and $1 R_{\odot}$.

\section{Summary and conclusions}

We performed a detailed spectral analysis of the magnetic HesdO J0809-2627 and used a simple homogeneous model for the magnetic field to derive a mean magnetic field strength of $353 \pm 10 \mathrm{kG}$ at an inclination of $\psi=64 \pm 25^{\circ}$. Apart from its strong magnetic field, J0809-2627 has a super-solar helium abundance at $\log n(\mathrm{He}) / n(\mathrm{H})=+0.28 \pm 0.10$, which is low compared to typical non-magnetic He-sdO stars. Its effective temperature, $T_{\text {eff }}=44900 \pm 1000 \mathrm{~K}$, and surface gravity, $\log g=$ $5.93 \pm 0.15$, are not unusual. The He-sdO is nitrogen-rich and seems to be carbon- and oxygen-poor, although only upper limits could be derived for $\mathrm{C}$ and $\mathrm{O}$. We combined astrometry, photometry, and spectroscopy to derive stellar parameters of $R=0.184 \pm 0.011 R_{\odot}, L=123_{-16}^{+19} L_{\odot}$, and $M=0.93_{-0.30}^{+0.44} M_{\odot}$. Placing it in the Hertzsprung-Russell diagram, J0809-2627 is located on the helium main sequence, which implies that it has completely lost any hydrogen envelope.

The TESS light curve of J0809-2627 does not show significant variability (see Appendix A.2). Assuming a radius of $0.184 R_{\odot}$ and a rotational velocity of less than $40 \mathrm{~km} \mathrm{~s}^{-1}$, a magnetic spot would be expected to result in a modulation at a period of more than $5.6 \mathrm{~h}$. The observed lack of photometric variability may be caused by a low inclination of the rotational axis, although a low inclination of the magnetic field axis seems to be excluded by spectroscopy. However, many magnetic main sequence stars are oblique rotators, which means that their magnetic axis is not aligned with their rotational axis (Landstreet \& Borra 1978). Photometric observations that are more sensitive than the $\approx 0.42 \mathrm{ppt}$ detection limit achieved by the current TESS data would help to further constrain the presence of magnetic spots.

In summary, the detection of the strong magnetic field of J0809-2627, its unusually high mass, its chemical composition, its slow projected rotation, and the lack of evidence for a close stellar companion provide overwhelming evidence for J08092627 being the result of a double-degenerate merger that produced a stably helium-burning star. Nonetheless, radial velocity monitoring still has to be done to exclude a close binary nature $^{2}$. As noted by Schwab (2018), magneto-hydrodynamic simulations would be required for suitable double He-WD mergers or He-WD+HeCO-WD mergers, similar to those performed for CO-WD mergers by Ji et al. (2013) and Zhu et al. (2015).

The double He-WD and He-WD+HeCO-WD merger scenarios are also both viable for the more common non-magnetic HesdO stars as well. Therefore, the question remains as to why the vast majority of He-sdO stars are apparently non-magnetic while J0809-2627 has a strong magnetic field. One possibility would

2 He-sdOs with masses as high as that of J0809-2627 or more could also form from intermediate mass stars that had their envelope stripped by a compact companion (Götberg et al. 2018). However, the stripping process is unlikely to generate a strong magnetic field, so the magnetic field would need to be primordial or generated during the red giant evolution of the progenitor. In addition, the stripped stars of Götberg et al. (2018) are predicted to be much more luminous than J0809-2627. 
be that the surface magnetic field observed for J0809-2627 is not stable and weakens quickly after a merging event.

While our simple homogeneous model for the magnetic field generally results in good fits for most lines in the X-shooter spectrum of J0809-2627, the magnetic field geometry might be more complicated in reality. Spectropolarimetric follow-up observations would provide more information about the magnetic field geometry. The current X-shooter spectrum excludes projected rotation velocities of more than about $40 \mathrm{~km} \mathrm{~s}^{-1}$, which is still consistent with rotation velocities predicted for some double He-WD merger remnants (Schwab 2018). Higher-resolution monitoring would further constrain this value. Ultraviolet observations would allow us to improve on the determination of abundances of carbon and oxygen, for which only upper limits are available at present, and would also provide abundance measurements for many more metals, including the iron group. A complete abundance pattern is crucial for establishing the composition of the accreted material.

Acknowledgements. We thank the referee, John Landstreet, for his very useful comments. We thank Ylva Götberg for discussions on stripped massive stars and Max Pritzkuleit for his support with the HOTFUSS project. UH and MD acknowledge funding by the Deutsche Forschungsgemeinschaft (DFG) through grants IR190/1-1, HE1356/70-1 and HE1356/71-1. IP acknowledges support from the UK's Science and Technology Facilities Council (STFC), grant ST/T000406/1. AGI acknowledges support from the Netherlands Organisation for Scientific Research (NWO). SJ acknowledges funding from the Netherlands Organisation for Scientific Research (NWO), as part of the Vidi research program BinWaves (project number 639.042.728, PI: de Mink). This research has made use of NASA's Astrophysics Data System. Based on observations made with ESO Telescopes at the La Silla Paranal Observatory under programme ID 105.206H.001 and observations with the Isaac Newton Telescope (under programme ID ING.NL.19B.005) operated on the island of La Palma by the Isaac Newton Group of Telescopes in the Spanish Observatorio del Roque de los Muchachos of the Instituto de Astrofísica de Canarias. This paper includes data collected by the TESS mission. Funding for the TESS mission is provided by the NASA's Science Mission Directorate. This work has made use of data from the European Space Agency (ESA) mission Gaia (https://www . cosmos.esa.int/gaia), processed by the Gaia Data Processing and Analysis Consortium (DPAC, https://www.cosmos.esa.int/web/gaia/dpac/ consortium). Funding for the DPAC has been provided by national institutions, in particular the institutions participating in the Gaia Multilateral Agreement.

\section{References}

Aller, A., Lillo-Box, J., Jones, D., Miranda, L. F., \& Barceló Forteza, S. 2020, A\&A, 635, A128

Bagnulo, S., \& Landstreet, J. D. 2020, A\&A, 643, A134

Bagnulo, S., \& Landstreet, J. D. 2021, MNRAS, 507, 5902

Bagnulo, S., Fossati, L., Landstreet, J. D., \& Izzo, C. 2015, A\&A, 583, A115

Bailer-Jones, C. A. L., Rybizki, J., Fouesneau, M., Mantelet, G., \& Andrae, R. 2018, AJ, 156, 58

Balona, L. A., Handler, G., Chowdhury, S., et al. 2019, MNRAS, 485, 3457

Condon, E. U., \& Shortley, G. H. 1935, The Theory of Atomic Spectra

Cutri, R. M., Skrutskie, M. F., van Dyk, S., et al. 2003, VizieR Online Data Catalog: II/246

Denis, C. 2005, VizieR Online Data Catalog: The DENIS database

Dorsch, M., Latour, M., \& Heber, U. 2019, A\&A, 630, A130
Dorsch, M., Latour, M., Heber, U., et al. 2020, A\&A, 643, A22

Dorsch, M., Jeffery, C. S., Irrgang, A., Woolf, V., \& Heber, U. 2021, A\&A, 653, A120

Drlica-Wagner, A., Carlin, J. L., Nidever, D. L., et al. 2021, ApJS, 256, 2

El-Badry, K., Rix, H.-W., \& Heintz, T. M. 2021, MNRAS, 506, 2269

Feinstein, A. D., Montet, B. T., Foreman-Mackey, D., et al. 2019, PASP, 131, 094502

Fitzpatrick, E. L., Massa, D., Gordon, K. D., Bohlin, R., \& Clayton, G. C. 2019, ApJ, 886, 108

Gaia Collaboration (Prusti, T., et al.) 2016, A\&A, 595, A1

Gaia Collaboration (Brown, A. G. A.) 2021, A\&A, 649, A1

García-Berro, E., Lorén-Aguilar, P., Aznar-Siguán, G., et al. 2012, ApJ, 749, 25

Garstang, R. H., \& Kemic, S. B. 1974, Ap\&SS, 31, 103

Geier, S., \& Heber, U. 2012, A\&A, 543, A149

Geier, S., Kupfer, T., Heber, U., et al. 2015, A\&A, 577, A26

Geier, S., Raddi, R., Gentile Fusillo, N. P., \& Marsh, T. R. 2019, A\&A, 621, A38

Götberg, Y., de Mink, S. E., Groh, J. H., et al. 2018, A\&A, 615, A78

Hall, P. D., \& Jeffery, C. S. 2016, MNRAS, 463, 2756

Han, Z., Podsiadlowski, P., Maxted, P. F. L., Marsh, T. R., \& Ivanova, N. 2002, MNRAS, 336, 449

Han, Z., Podsiadlowski, P., Maxted, P. F. L., \& Marsh, T. R. 2003, MNRAS, 341, 669

Heber, U. 2009, ARA\&A, 47, 211

Heber, U. 2016, PASP, 128, 082001

Heber, U., Geier, S., \& Gaensicke, B. 2013, Eur. Phys. J. Web Conf., 43, 04002

Heber, U., Irrgang, A., \& Schaffenroth, J. 2018, Open Astron., 27, 35

Hönl, H. 1925, Z. Phys., 31, 340

Hubeny, I., \& Lanz, T. 2017a, ArXiv e-prints [arXiv:1706.01859]

Hubeny, I., \& Lanz, T. 2017b, ArXiv e-prints [arXiv:1706.01935]

Hubeny, I., \& Lanz, T. 2017c, ArXiv e-prints [arXiv:1706.01937]

Iben, Jr., I. 1990, ApJ, 353, 215

Irrgang, A., Geier, S., Heber, U., et al. 2021, A\&A, 650, A102

Jeffery, C. S., Ramsay, G., Naslim, N., et al. 2013, MNRAS, 429, 3207

Ji, S., Fisher, R. T., García-Berro, E., et al. 2013, ApJ, 773, 136

Justham, S., Podsiadlowski, P., \& Han, Z. 2011, MNRAS, 410, 984

Kawka, A., \& Vennes, S. 2011, A\&A, 532, A7

Landstreet, J. D., \& Borra, E. F. 1978, ApJ, 224, L5

Landstreet, J. D., Bagnulo, S., Fossati, L., Jordan, S., \& O’Toole, S. J. 2012, A\&A, 541, A100

Lindegren, L., Bastian, U., Biermann, M., et al. 2021, A\&A, 649, A4

Lorén-Aguilar, P., Isern, J., \& García-Berro, E. 2009, A\&A, 500, 1193

Magnier, E. A., Schlafly, E. F., Finkbeiner, D. P., et al. 2020, ApJS, 251, 6

Martin, B., \& Wickramasinghe, D. T. 1981, MNRAS, 196, 23

Mathys, G., Hubrig, S., Mason, E., et al. 2012, Astron. Nachr., 333, 30

Moehler, S., Modigliani, A., Freudling, W., et al. 2014, A\&A, 568, A9

Momany, Y., Zaggia, S., Montalto, M., et al. 2020, Nat. Astron., 4, 1092

Onken, C. A., Wolf, C., Bessell, M. S., et al. 2019, PASA, 36, e033

Paczyński, B. 1971, Acta Astron, 21, 1

Pelisoli, I., Vos, J., Geier, S., Schaffenroth, V., \& Baran, A. S. 2020, A\&A, 642, A180

Randall, S. K., Bagnulo, S., Ziegerer, E., Geier, S., \& Fontaine, G. 2015, A\&A, 576, A65

Riello, M., De Angeli, F., Evans, D. W., et al. 2021, A\&A, 649, A3

Schindewolf, M., Németh, P., Heber, U., et al. 2018, A\&A, 620, A36

Schlafly, E. F., Green, G. M., Lang, D., et al. 2018, ApJS, 234, 39

Schneider, F. R. N., Ohlmann, S. T., Podsiadlowski, P., et al. 2019, Nature, 574 211

Schwab, J. 2018, MNRAS, 476, 5303

Stroeer, A., Heber, U., Lisker, T., et al. 2007, A\&A, 462, 269

Tremblay, P. E., Fontaine, G., Freytag, B., et al. 2015, ApJ, 812, 19

Vos, J., Pelisoli, I., Budaj, J., et al. 2021, A\&A, 655, A43

Webbink, R. F. 1984, ApJ, 277, 355

Wickramasinghe, D. T., \& Martin, B. 1986, MNRAS, 223, 323

Yoon, S. C., Podsiadlowski, P., \& Rosswog, S. 2007, MNRAS, 380, 933

Zhang, X., \& Jeffery, C. S. 2012, MNRAS, 419, 452

Zhu, C., Pakmor, R., van Kerkwijk, M. H., \& Chang, P. 2015, ApJ, 806, L1 


\section{Appendix A: Observations}

\section{A.1. Spectroscopy}

Details of the spectroscopic observations are listed in Table A.1.

Table A.1. Dates and exposure times for the spectroscopic observations of J0809-2627.

\begin{tabular}{lcc}
\hline \hline Spectrograph & Mid exposure BJD & Exposure time (s) \\
\hline INT/IDS & 2458837.6695 & 750 \\
INT/IDS & 2458837.6798 & 750 \\
VLT/X-shooter & 2459314.5691 & 1800 \\
\hline
\end{tabular}

\section{A.2. TESS}

J0809-2627 was in the TESS field during sectors 7, 8, and 34 The star was not targeted for short-cadence observations, but the full-frame images are available. They have an integration time of $30 \mathrm{~min}$ for sectors 7 and 8 , and $10 \mathrm{~min}$ for sector 34 . The total time span of the light curve is 760 days. We performed the photometry of all available images using eleanor (Feinstein et al. 2019). As the field is relatively crowded (see Fig. A.1), we used the small aperture option, which limits aperture size to 8 pixels ${ }^{3}$. We then carried out the Fourier transform of all the data up to the Nyquist frequency, as shown in Fig. A.2. In order to determine a detection limit, we randomly assigned one of the measured fluxes to each measured time and recalculated the Fourier transform a thousand times, each time recording the maximum amplitude. We selected the maximum overall value as the detection limit; as it happens once every 1000 runs, this corresponds to a false-alarm probability of $1 / 1000$. No peaks are detected above this threshold; therefore, we find no periodic variability for periods between $\approx 20$ min and $\approx 380$ days down to a detection limit of $\approx 0.42 \mathrm{ppt}$. It is important to emphasise, however, that the large pixel size can result in contamination from nearby constant stars, which can hide any variability. Follow-up with better image resolution is encouraged.

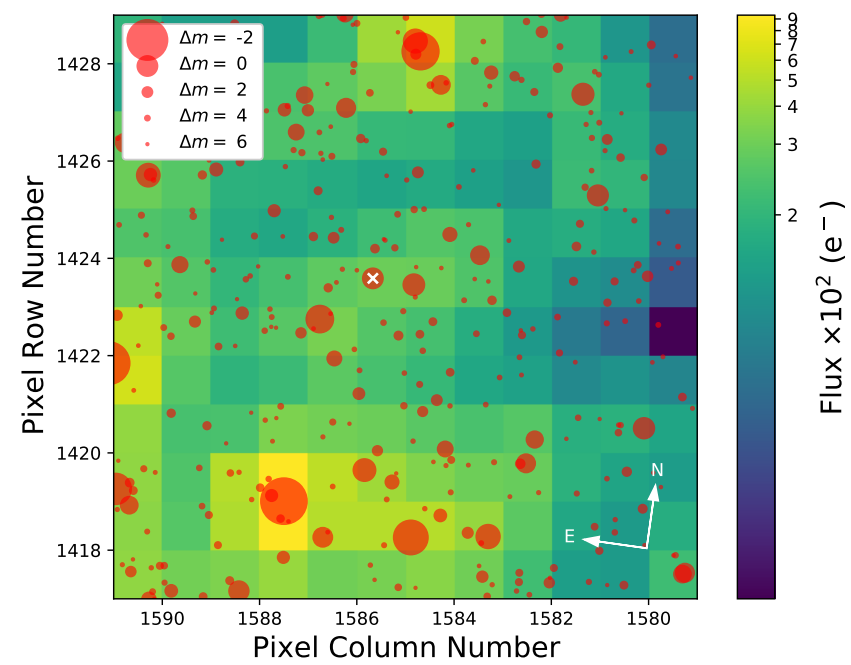

Fig. A.1. Image from TESS sector 7 created with tpfplotter (Aller et al. 2020) showing the location of J0809-2627 (white cross) and other stars with a magnitude difference of six or less (red circles).

\footnotetext{
The TESS pixel size is 21 ".
}

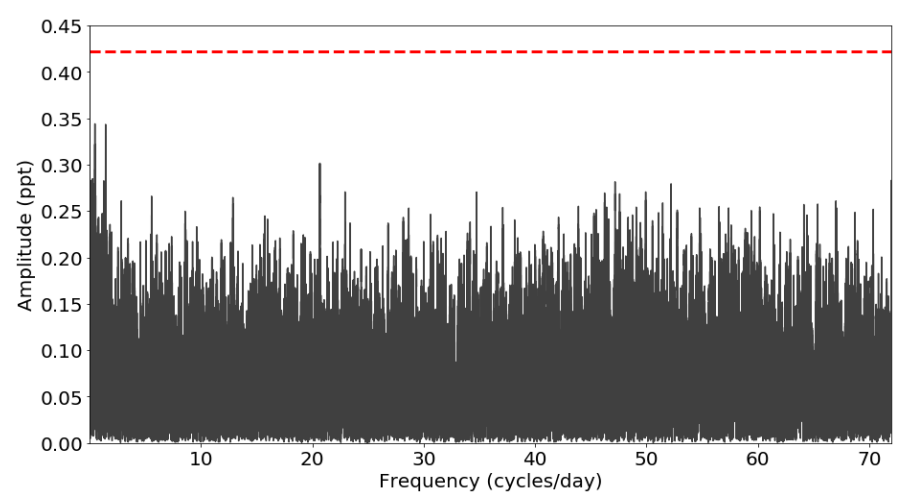

Fig. A.2. Fourier transform of the TESS data. The amplitude is shown in ppt. The adopted detection limit of $0.42 \mathrm{ppt}$ is shown as a dashed red line, and no peaks are detected above this limit.

\section{Appendix B: Linear Zeeman effect}

We assume LS coupling for all metal line transitions given that the heaviest observed element is sulphur. An LS coupling state is described by its orbital angular momentum, $L$, its spin angular momentum, $S$, and its total angular momentum, $J$. For each included line, the upper and lower LS states were split into $2 J+1$ components with magnetic quantum numbers from $m=-J$ to $m=+J$. Only transitions with $\Delta m=0$ or \pm 1 were considered.

For a transition between lower and upper states with magnetic quantum numbers $m_{\mathrm{l}}$ and $m_{\mathrm{u}}$, the wavelength shift with respect to the rest wavelength at zero magnetic field, $\lambda_{0}$, is given as

$\Delta \lambda=\frac{e B \lambda_{0}^{2}}{4 \pi m_{e} c^{2}} \cdot\left(m_{1} g_{1}-m_{\mathrm{u}} g_{\mathrm{u}}\right)$,

where $e$ is the elementary charge, $B$ is the magnetic field strength, $m_{e}$ is the electron mass, $c$ is the speed of light, and $g_{1}$ and $g_{\mathrm{u}}$ are the Landé $g$-factors for the lower and upper level.

In the case of LS coupling, the Landé $g$-factor is given as

$g=1+\frac{J(J+1)+S(S+1)-L(L+1)}{2 J(J+1)}$.

Transitions between levels of hydrogen and singly ionised helium represent special cases in this model for the magnetic field because these levels are sufficiently described by a main quantum number, $n$. For these levels, as well as neutral helium, the Landé $g$-factors were set to unity.

Table B.1. Relative intensities for the $\pi$ and $\sigma$ components following Hönl (1925). The quantum numbers $J$ and $m$ refer to the initial level, and $\Delta J=J_{\text {final }}-J_{\text {initial }}$. We note the difference from Eq. (5) of Kawka \& Vennes (2011), which contains a sign error.

\begin{tabular}{ccr}
\hline \hline$\Delta J$ & $\pi$ & $\pm \sigma$ \\
\hline 0 & $m^{2}$ & $\frac{1}{4}(J \mp m)(J \pm m+1)$ \\
+1 & $(J+1)^{2}-m^{2}$ & $\frac{1}{4}(J \pm m+1)(J \pm m+2)$ \\
-1 & $J^{2}-m^{2}$ & $\frac{1}{4}(J \mp m)(J \mp m-1)$ \\
\hline
\end{tabular}

Analytic expressions for the relative intensities of the components of any linear Zeeman multiplet were first presented by Hönl (1925). They are summarised in Table B.1. Unlike the Landé $g$-factors, these relative intensities depend only on $J$ and $m$, and are therefore valid in any coupling scheme. 
The relative intensity $(I)$ of the central $(\pi)$ and displaced $(\sigma)$ components depends on the angle $(\psi)$ between the magnetic field axis and the line of sight. The angular dependence given by Condon \& Shortley (1935) is for the $\pi$ (central) component

$$
\frac{I(\psi)}{I\left(90^{\circ}\right)}=\sin ^{2} \psi
$$

and for the $\sigma$ components

$$
\frac{I(\psi)}{I\left(90^{\circ}\right)}=1+\cos ^{2} \psi
$$

\section{Appendix C: Mass, radius, luminosity, and stellar population characteristics}

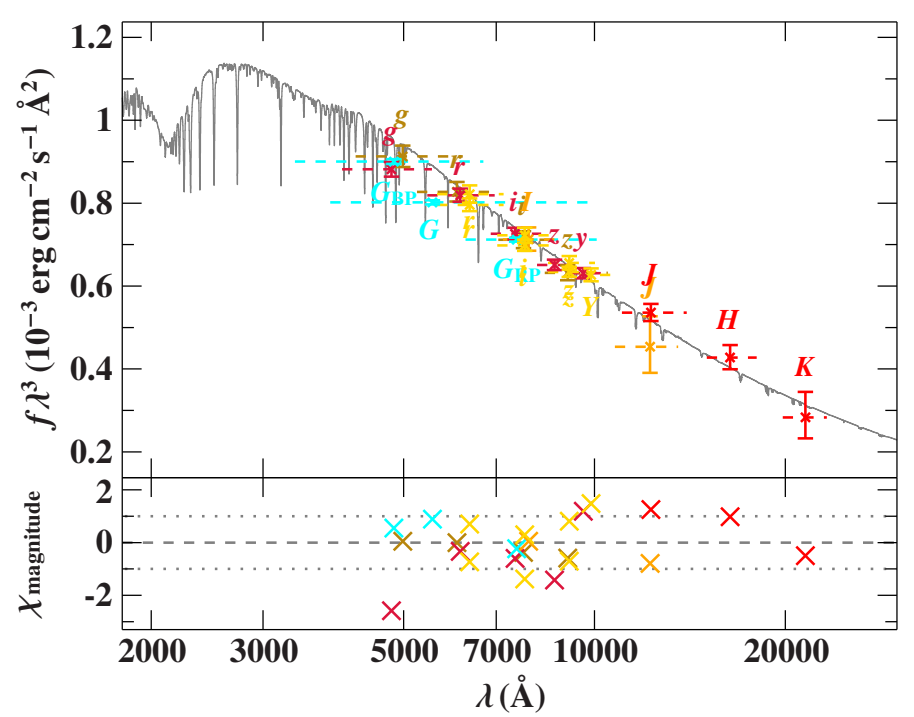

Fig. C.1. SED fit for J0809-2627. The grey line shows the final model of J0809-2627, and filter-averaged flux measurements are indicated by dashed horizontal lines. Photometric surveys are identified by colour codes: Pan-STARRS (dark red; Magnier et al. 2020), SkyMapper (dark gold; Onken et al. 2019), Gaia (cyan; Riello et al. 2021), DECam (gold; Schlafly et al. 2018; Drlica-Wagner et al. 2021), DENIS (orange; Denis 2005), and 2MASS (red; Cutri et al. 2003).

We constructed the SED of J0809-2627 by collecting photometric measurements from several surveys. Two SED fits were then performed using the same grid as for the spectroscopic analysis, with $T_{\text {eff }}$ either as a free parameter or fixed to the value derived by the spectroscopic fit (for details, see Heber et al. 2018; Irrgang et al. 2021). The latter approach is useful because $T_{\text {eff }}$ $\gtrsim 35000 \mathrm{~K}$ are not well constrained from optical and infrared magnitudes alone. The surface gravity and helium abundance were always fixed to spectroscopic values, and the angular diameter $\Theta$ was always a free parameter. Interstellar extinction was accounted for using the reddening law of Fitzpatrick et al. (2019) with the colour excess $E_{44-55}$ as a free parameter. An extinction parameter of $R(55)=3.02$ was assumed.
Table C.1. SED fit and stellar parameters. The stellar parameters result from the SED fit with a prescribed $T_{\text {eff }}=44900 \pm 1000 \mathrm{~K}$. The mode and the highest density interval of each quantity are given for $1 \sigma$ probability (see Bailer-Jones et al. 2018).

\begin{tabular}{lr}
\hline \hline & SED fit \\
\hline$T_{\text {eff }}(\mathrm{K})$ & $47000_{-3000}^{+11000}$ \\
\hline $\log \Theta(\mathrm{rad})$ & $-11.246_{-0.004}^{+0.005}$ \\
$E_{44-55}(\mathrm{mag})$ & $0.073_{-0.004}^{+0.004}$ \\
Radius $R=\Theta /(2 \varpi)$ & $0.184_{-0.0110}^{+0.011} R_{\odot}$ \\
Mass $M=g R^{2} / G$ & $0.93_{-0.30}^{+0.44} M_{\odot}$ \\
Luminosity $L / L_{\odot}=\left(R / R_{\odot}\right)^{2}\left(T_{\text {eff }} / T_{\text {eff, } \odot}\right)^{4}$ & $123_{-16}^{+19}$ \\
\hline
\end{tabular}

The angular diameter derived from the SED (with fixed $T_{\text {eff }}$ ) was combined with the parallax measurement provided by Gaia Early Data Release 3 (EDR3; Gaia Collaboration 2016, 2021) and the spectroscopic surface gravity to estimate the stellar parameters mass, radius, and luminosity for J0809-2627. Here, the parallax measurement, $\varpi=0.68 \pm 0.04$ mas, was corrected for its zero point offset following Lindegren et al. (2021), and the corresponding uncertainty was inflated using the function of El-Badry et al. (2021). The SED fit and stellar parameters are summarised in Table C.1. Figure C.1 shows the SED fit with $T_{\text {eff }}$ fixed to the spectroscopic value.

Because J0809-2627 is close to the Galactic disk, we expect it to belong to the thin disk stellar population I. This can be confirmed by calculating its current Galactic space velocity vector from the radial velocity (assumed to be nonvariable), parallax, and proper motions provided by Gaia EDR3 (Gaia Collaboration 2021). The resulting vector $(U, V, W)=$ $(-17.3 \pm 2.0,230.1 \pm 2.7,7.1 \pm 0.8) \mathrm{km} \mathrm{s}^{-1}$, where $U$ is measured towards the Galactic centre, $V$ in the direction of Galactic rotation, and $W$ perpendicular to the disc, is consistent with a membership of the thin disk population.

\section{Appendix D: Spectral comparisons}

Here we show spectral comparisons for the X-shooter spectra of J0809-2627, specifically the UVB and VIS spectral arms up to $7400 \AA$. The strongest lines are labelled at their position for zero magnetic field. Spectral regions that are not well reproduced by our models were excluded from the fit. This includes several metal lines, as well as some neutral helium lines. Especially narrow He I lines from the triplet $(S=1)$ system, such as He I $5876 \AA$, and lines with strong forbidden components, such as He I 4472, $4922 \AA$, are not well modelled. This is to be expected given the simple nature of our model for the magnetic field, as well as the lack of helium broadening tables in the presence of a magnetic field. The C III lines at $4070 \AA$ are poorly modelled due to limitations of the TLUSTY model ion. Telluric lines were removed using the grid of transmission spectra provided by Moehler et al. (2014). 
M. Dorsch et al.: Discovery of a highly magnetic He-sdO
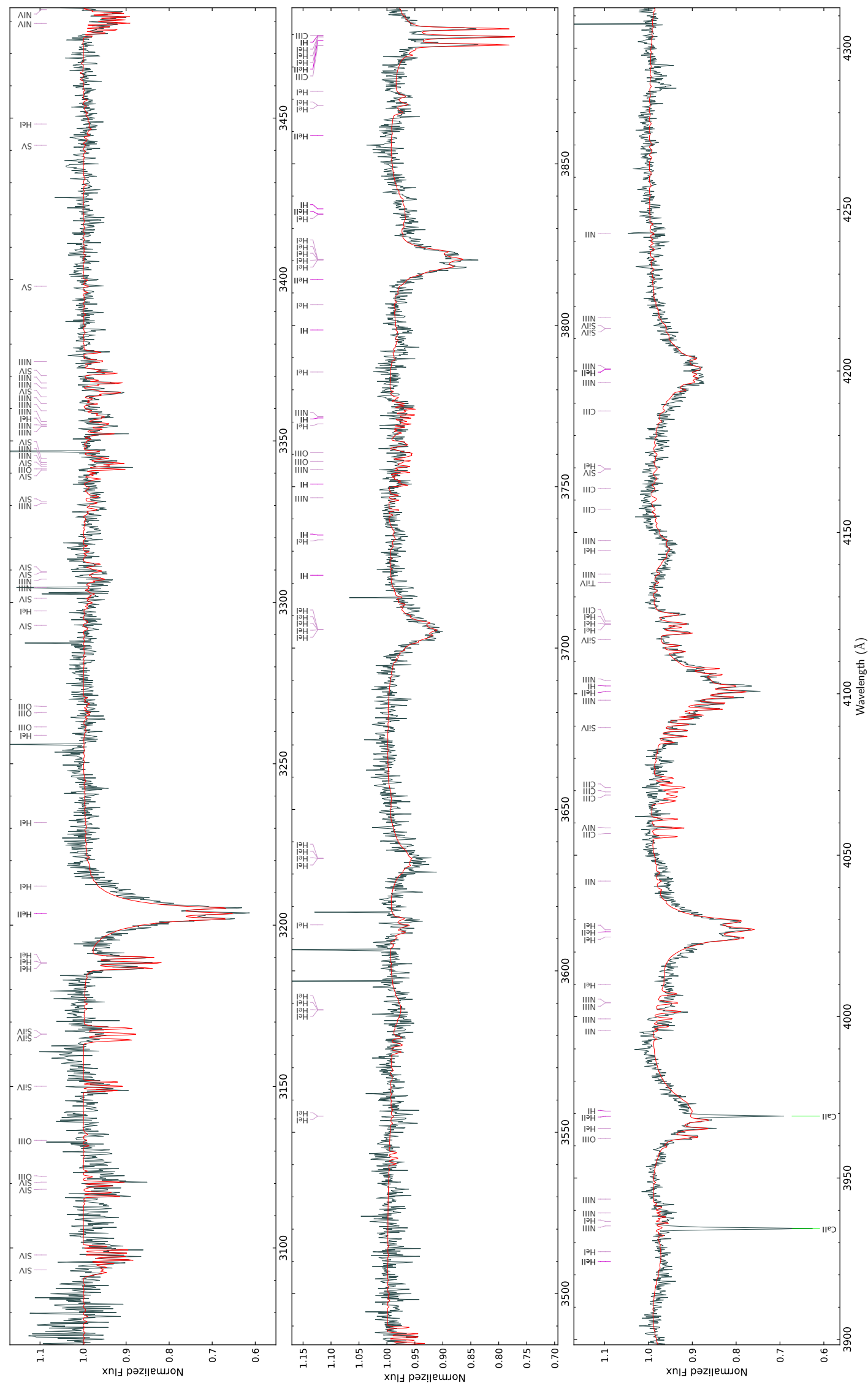

Fig. D.1. X-shooter spectrum of J0809-2627 (grey) and the best-fit model (red). 
A\&A 658, L9 (2022)

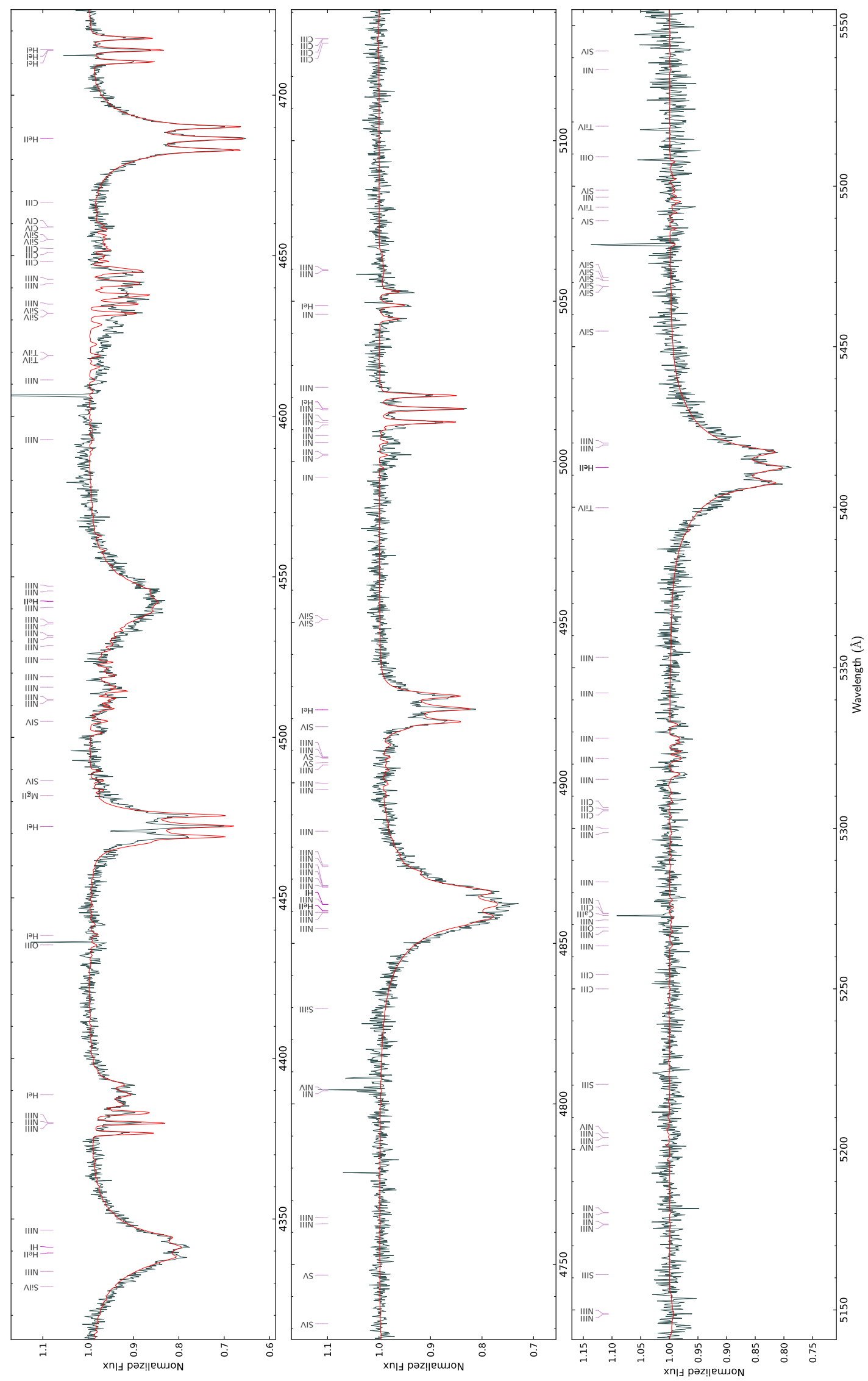

Fig. D.1. continued. 
M. Dorsch et al.: Discovery of a highly magnetic He-sdO

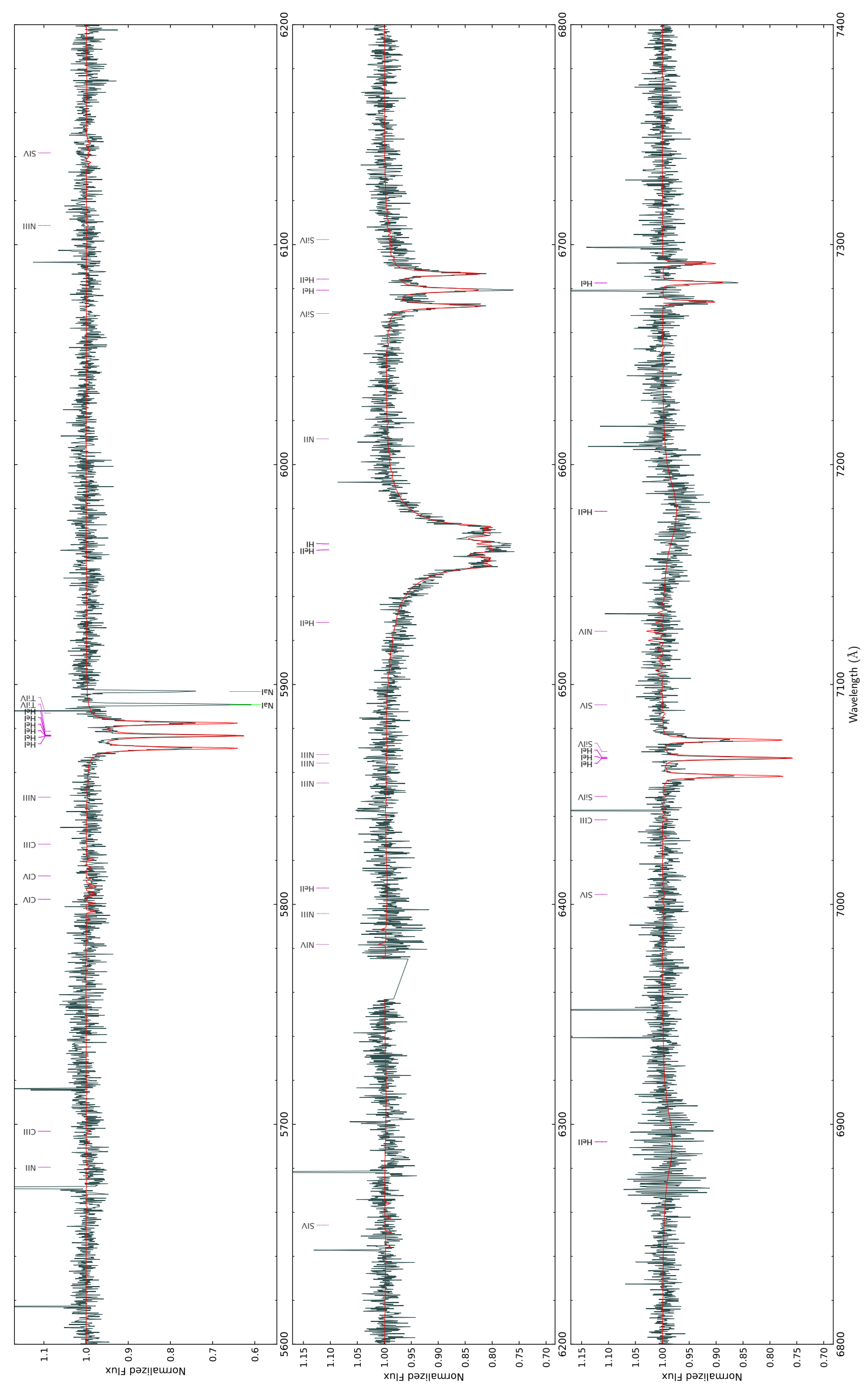

Fig. D.1. continued. 